\title{
Design of Electromechanical Temperature early warning system based on BP neural network
}

\author{
Jun Sheng \\ Pingdingshan Industrial College Of Technology; Hennan Pingdingshan 467001, China \\ shengjun@163.com
}

\begin{abstract}
Keywords: Electromechanical, Temperature early warning, Equipments Secure Management, BP
\end{abstract} neural network

\begin{abstract}
For the current situation that the coal mine accidents occurred frequently, especially the fact that most major accidents ware caused by electromechanical fires, this paper analyses the cause of the accident caused by electromechanical, proposes the temperature monitoring and early warning management system based on the BP neural network. Accordance the property of BP neural network can approximate any nonlinear function to analysis data effectively. This system can provide comprehensive, timely and accurate monitoring for the electromechanical, and predict the state of device temperature in a certain time to preventing accidents. At the same time, it can provide reliable and effective decision support for the security management work.
\end{abstract}

\section{Introduction}

In order to protect electromechanical safe, efficient and normal operation, we must monitor continuously. Changes of electromechanical operation temperature can response the degree of line aging and load commendably[1]. For the temperature monitoring way of traditional device and problems in reality, this paper using the latest optical fiber technology to collecting the devices temperature data and using the advantage of computers and networks to monitoring equipment running status comprehensive and timely. This system can avoid the accident caused by sudden equipment failure effectively[2].

This paper according to the existing coal mine electromechanical safety management, establishing the temperature warning management system based on the BP neural network. Through the application of temperature alarm management system, we can not only bring reliable protection for the safe operation of electromechanical, but also reducing the number of electromechanical manager and the single workload[3]. At the same time, using this system can avoid security risks due to the error operational in human Monitoring work.

\section{Design of BP neural network algorithm}

BP algorithm. According to the basic idea of BP algorithm, by calculate minimum of error functions to implement network training. BP algorithm will be described by the mathematical expression[4].

Assumed to have m layer neural network, the number of neurons in the out put layer is $X$. then the sum of the $i$-th of $k$ layer neuron input is $U_{i}{ }^{k}$, and the sum of the $i$-th of $k$ layer neuron output is $X_{i}{ }^{k}$. Then weight of the $j$-th neuron in the $k$-1 layer and the $i$-th neuron in the $k$ layer is $W_{i j}$. Excitation function is $f$. Expressions are as follows[5]:

$$
\begin{aligned}
& X_{i}{ }^{k}=f\left(U_{i}{ }^{k}\right) \\
& U_{i}{ }^{k}=\sum_{j} W_{i j} X_{j}{ }^{k-1}
\end{aligned}
$$

Set error function is $e$, then the quadratic sum of the desired output and the actual output is error function: 


$$
e=\frac{1}{2} \sum_{i}\left(X_{i}^{m}-Y_{i}\right)^{2}
$$

$\Delta W_{i j}$ is the change amount of weight $W_{i j}$ :

$$
\Delta W_{i j}=-\eta \frac{\partial e}{\partial W_{i j}}
$$

Then we can get the formula:

$$
d_{i}^{k}=X_{i}^{k}\left(1-X_{i}^{k}\right) \sum_{l} W_{i j} d_{l}^{k+1}
$$

Compared $X_{i}{ }^{m}$ and $Y_{i}$, if they are not equal, then get a error signal $e$, Continue reverse modify the weights according to the following formula:

$$
\Delta W=\sum_{j} W_{i j} X_{j}^{k-1}
$$

Through repeated training samples and amended weights continuous to eliminate errors eventually. From the above formula can also be informed that with the number of network layer increasing, the computing increases and resulting in slow convergence[6].

Improved BP algorithm. The way to make learning rate has adaptive learning regular is, by calculate the correction value of weight, Verify that weather the error function reduced, if so, explains the learning rate value select smaller, otherwise, we should select the value decrease the learning rate. An adaptive learning rate formula widely used is[7]:

$$
\eta(k+1)=\left\{\begin{array}{c}
1.05 \eta(k), \operatorname{SSE}(k+1)<\operatorname{SSE}(k) \\
0.7 \eta(k), \operatorname{SSE}(k+1)>1.04 \operatorname{SSE}(k) \\
\eta(k), \text { other }
\end{array}\right.
$$

Through continuous experiment, the learning rate can be increased in the case of network convergence in order to reduce the time to study. When the learning rate is too big to make the network convergence, it should be reduced until it can be converged in time.

Algorithm flow chart shown in Figure 1: 


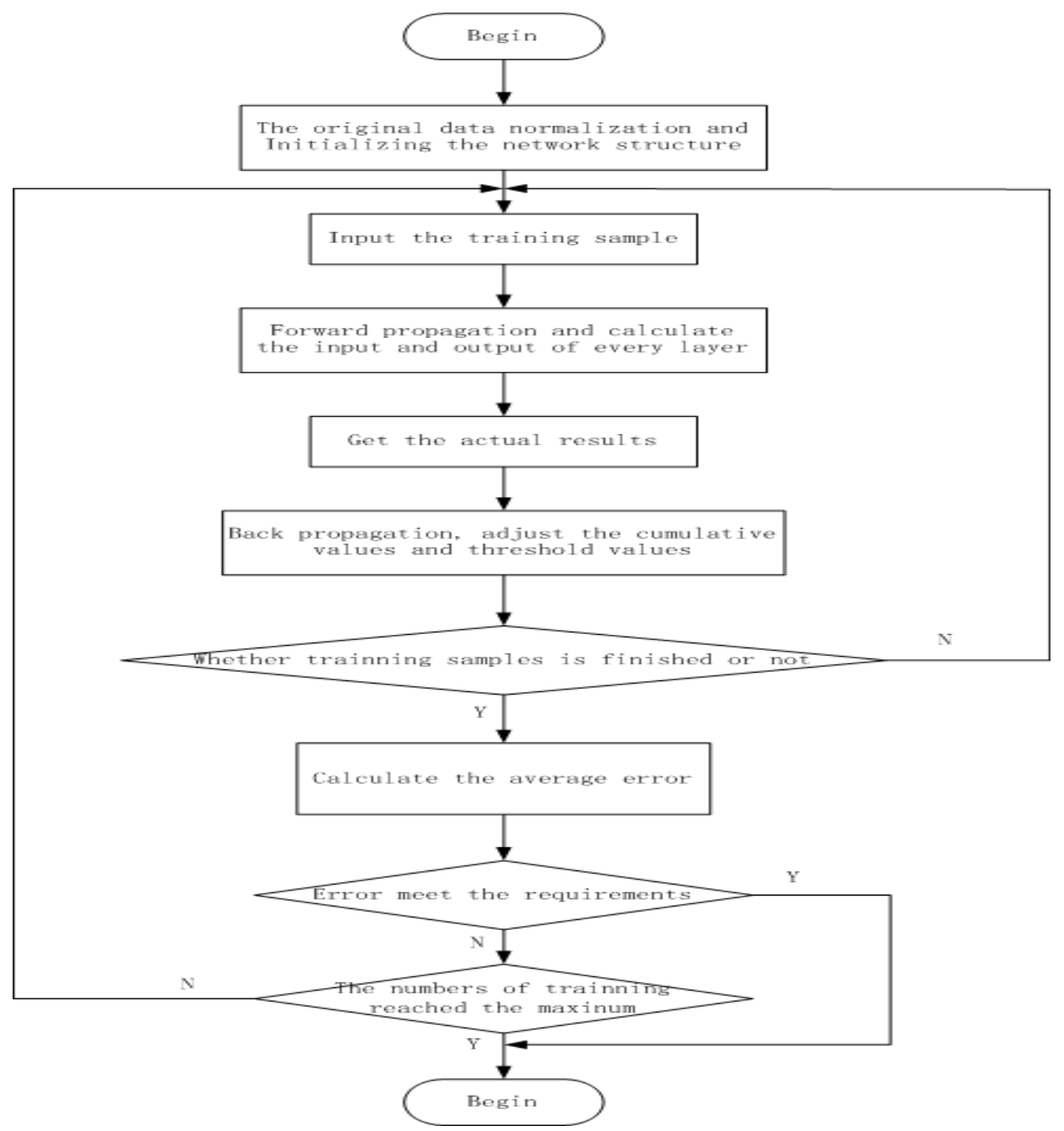

Fig.1 Flow of BP algorithm in this paper

\section{Experimental results and analysis}

In this paper, the experimental data is taken from a database record of a mine electrical and mech anical equipment of substation switchgear temperature monitoring system. Through the analysis an d selecting of experimental data, a specific temperature measuring point can be selected from numer ous temperature measuring points in the BP neural network model of switch cabinet temperature pre diction. Then the temperature data can be collected every two hour on the hour, and every day 12 te mperature data can be obtained. Data is collected in the three hours will be used for the training sam ple, and the day data is collected in the fourth day is used for the predict sample, and it will be used for error analysis in the last. The data sample after normalization is show in the table 1.

Tab.1 Data sample after normalization

\begin{tabular}{llll}
\hline & Day1 & Day2 & Day3 \\
\hline 1 & 0.9723 & 0.7354 & 0.9924 \\
3 & 0.8812 & 0.5782 & 0.9347 \\
5 & 0.9439 & 0.6245 & 1 \\
7 & 0.7342 & 0.5363 & 0.4256 \\
9 & 0.6425 & 0.9783 & 0.7356 \\
11 & 1 & 0.4683 & 0.8245 \\
13 & 0.5385 & 1 & 0.5265 \\
15 & 0.4678 & 1 & 0.9363 \\
17 & 0.4759 & 0.6337 & 0.6356 \\
19 & 0.9647 & 0.7467 & 0.8356 \\
21 & 0.4683 & 0.9467 & 1 \\
23 & 1 & 0.5367 & 0.6298 \\
\hline
\end{tabular}


The comparison of actual sample curve and prediction curve showed in figure 2.

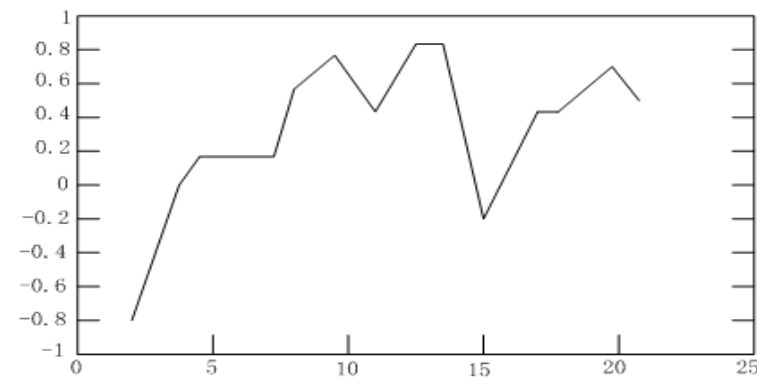

(a) Real sample curve changes

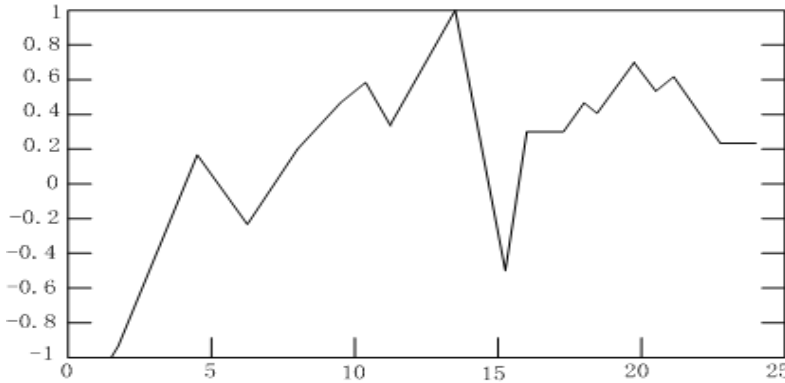

(b) Predicted sample curve changes

Fig. 2 The comparison of actual sample curve and prediction curve

As it can be seen, it is successful for predicting temperature by using the model. Though BP neural network model to forecast temperature warning electromechanical devices is very feasible. Electromecchanical managers can do the Network simulation training and testing to get the future temperature charts, to discover possible equipment failure, effectively prevent sudden accidents and reached the warning purpose.

\section{Conclusions}

This paper analyses the temperature data thought the BP neural network, setting the appropriate network in the system and get the corresponding predictions. Through the application of temperature alarm management system, we can get the operational status of equipment. Avoid sudden stops due to maintenance work resulting in the production. Using this system can avoid security risks due to the error operational in human Monitoring work.

\section{References}

[1] Han M.Han B. Xi J H.et al. Universal Learning Network And Its Application For Nonlinear System With Long Time Delay(Computers \&Chemical Engineering, 2006), p.13-20.

[2] Hassan.K. KhaliLNonlinear Systems-Electronic Engeering Public(China's industrial university press, China 2005), p.233-236. (In Chinese)

[3] Time E. Pringle \& Stephen D. Frost.The Absence of Rigor and The Failure of Implementation:Occupational Health and Safety in China(NSW Minerals Council, 2002).

[4] J.N.MurPhy. Coal Mine Health And Safety Research In The USA — The Achievements Of The US Bureauof Mines(Coal International, 1994), p.63-85.

[5] Y Chen.K Moore. V.B.ahl. Learning Feed Forward Control Using A Dilated B-spline Network: Frequency Domain Analysis And Design(IEEE Trans. Neural Netw, November 9-20,2004), Vol. 2, p.124.

[6] Hirasawa.K.Kim S.Hu J L.et al. Improvement Of Generalization Ability For Identifying Dynamical Systems By Using Universal Learning Networks(Neural Networks), Vol. 19 (2006) No. 4, p.487-499.

[7] Muriel PEREZ. Neural Networks Applications In Bankruptcy Forecasting: A State of the Art. 1999. 